\title{
Contextual conditioning during free-operant extinction: Unsignaled, signaled, and backward-signaled noncontingent food
}

\author{
A. G. BAKER \\ McGill University, Montreal, Quebec, Canada
}

\begin{abstract}
Three experiments were designed to study the effects of contextual conditioning on the extinction of instrumental leverpressing that had been reinforced on a random-interval schedule. In Experiment 1, noncontingent food retarded extinction, but signaling food delivery, a treatment that should reduce contextual conditioning, reduced the interference. Experiment 2 replicated the results of Experiment 1 and demonstrated that if the food preceded rather than followed the signal, the retardation of extinction was not reduced but was enhanced. In Experiment 3, noncontingent leverpressing was used to directly verify that the three treatments-forward signaling, noncontingent food, and backward signaling-differentially influenced contextual conditioning. Forward signaling produced the least, and backward signaling produced the most, contextual conditioning. This monotonic relationship between contextual conditioning and interference with extinction was used as evidence to support the argument that context-food associations are important in controlling instrumental responding.
\end{abstract}

There has been much elegant experimental analysis aimed at demonstrating that animals can represent the specific response-reinforcer association involved in instrumental conditioning (e.g., Colwill \& Rescorla, 1986). It has been claimed that they learn these relationships in propositional form (e.g., see Adams \& Dickinson, 1981; Dickinson \& Charnock, 1985). The purpose of the present experiments was to demonstrate that a more traditional associative factor is also important in the control of instrumental responding. Traditionally (e.g., see Spence, 1966), it has been argued that one of the important factors involved in energizing instrumental responding is the associations that are formed between the experimental context and the reinforcer. These associations might have motivational significance or they themselves might be part of the controlling stimuli that set the occasion for the response. In the present experiments, I studied the role of context-reinforcer associations in the maintenance of responding during the extinction of leverpressing that had been reinforced on a random-interval schedule.

The traditional method of weakening an instrumental or operant response is the extinction procedure, in which the opportunity to emit the response continues but the reinforcer is discontinued. In the 1960s, Rescorla (e.g., Rescorla, 1966) carried out a series of classical conditioning experiments that demonstrated that the crucial determinant

This research was supported by a Natural Sciences and Engineering Research Council of Canada operating grant. I would like to thank G. Hall, M. E. Bouton, and C. E. Haskins for their comments on various drafts. Correspondence concerning this article may be sent to $A$. $G$. Baker, Department of Psychology, McGill University, 1205 Dr. Penfield Dr., Montreal, Quebec H3A 1B1, Canada. of the conditioned response is the correlation between the conditional stimulus (CS) and the unconditional stimulus (US), and not temporal contiguity per se. This view suggests that a response may be weakened without eliminating the reinforcer simply by reducing the contingency between the response and the reinforcer. Rescorla and Skucy (1969) performed an experiment that confirmed this expectation. They initially trained rats to respond for food on a variable-interval schedule of reinforcement and then attempted to weaken the response either by removing the food (extinction) or by continuing to present the food at about the same frequency that it had been presented before but doing so independently of responding (noncontingent or "free" food). Free food reduced responding substantially, but did so more slowly than did extinction. Put another way, removing the contingency but continuing reinforcement retarded extinction. At about the same time, several other experimenters reported a similar interference with extinction (Boakes, 1973; Herrnstein, 1966; Herrnstein \& Morse, 1957; Neuringer, 1970; Spence, 1966).

It must be emphasized that although this result is consistent with the contingency or correlational viewpoint, it is also consistent with the older notion of temporal contiguity. The extinction procedure completely removes temporal contiguity, but free food only reduces it. As long as the animal is still responding, food is still contiguous with the response, although less so than with contingent food delivery. It is thus possible that removing the contingency or correlation between responding and food reduces responding only because it moderately reduces temporal contiguity and not because of the change in contingency. 
Rescorla and Skucy (1969) dealt with this possibility by including a control group in which free food was presented but in which there was a negative contingency. Any response occurring at about the time of a free-food delivery would delay the food by at least $5 \mathrm{sec}$. This procedure produced a rather rapid short-term drop in responding, probably because the high initial response rates eliminated most of the food, making the treatment much like normal extinction. Nevertheless, this negative-contingency group ultimately emitted a total number of responses equivalent to that of the noncontingent-food group.

Although Rescorla and Skucy's (1969) above demonstration weakened the case for response-reinforcer contiguity as the unitary explanation of the retardation of extinction caused by free food, they did not suggest an alternative mechanism for this retardation. The simplest explanation of the interference is that it is a case of generalization decrement. Animals receiving the free-food treatment extinguish more slowly because it is more difficult to discriminate between free food and training than it is to discriminate between traditional extinction and training. This hypothesis is similar to Capaldi's (e.g., Capaldi, 1967) analysis of the partial reinforcement effect in extinction, which claims that during instrumental conditioning, responding comes under the control of the various stimulus properties of the experimental situation. These properties include the presence of both reinforcement and nonreinforcement. When food is removed during extinction, responding declines simply because removing the food changes the stimulus situation enough to reduce responding. No reference to the weakening of the responsereinforcer bond need be made. The initial responsereinforcer bond remains intact during extinction; responding declines because of generalization decrement.

The generalization-decrement hypothesis can not be taken seriously as a complete explanation of extinction, but it does have much to recommend it as one part of a hybrid explanation (see Mackintosh, 1974). One prediction that it does make is that maintaining free food during extinction should retard extinction to the extent that it maintains the stimulus properties found during training, and thus reduces generalization decrement.

A second alternative that will be tested here stems from an associative approach to instrumental conditioning. According to this view, responding is controlled not only by response-food associations, but also by the associations formed between the experimental context and food. During training, the context becomes conditioned and thus could come to control responding through at least two mechanisms: (1) the conditioned context has stimulus properties and the response comes at least partially under control of these properties, and (2) the context develops incentive properties that directly strengthen the response. The former mechanism is another version of the generalization-decrement hypothesis and the latter represents a more traditional associative view.

\section{EXPERIMENT 1}

If contextual conditioning is at least partially responsible for the retardation of extinction by free food, then any manipulation that reduces this conditioning should facilitate extinction. It has frequently been demonstrated (e.g., Baker, Mercier, Gabel, \& Baker, 1981; Grau \& Rescorla, 1984; Odling-Smee, 1978; for exceptions see Balsam, 1985; Rescorla, Durlach, \& Grau, 1985) that contextual conditioning may be reduced by providing a more temporally contiguous cue (a signal) for the reinforcer. If the reinforcer is signaled, contextual conditioning is overshadowed (see Pavlov, 1927; Kamin, 1969).

The present experiment provided a test of the assumption that if free food is signaled during extinction, this signaling should reduce contextual conditioning, and extinction should be facilitated to the extent that this conditioning controls responding. The experiment included three groups of rats. All three groups were initially trained to barpress for food on a random-interval schedule of reinforcement and, following this, three procedures were used to reduce responding. Group Extinction received the traditional extinction procedure in which food was no longer delivered. Group Free Food received no responsecontingent food during this period but did receive noncontingent food. There was no attempt to eliminate chance response-food pairings in this group. Finally, Group Signaled Food received the same treatment as Group Free Food except that each food presentation was signaled by a brief light.

\section{Method}

Subjects. Twenty-four male Long-Evans hooded rats that were obtained from Charles River (Canada) served as subjects. These rats were reduced to $80 \%$ of their initial weights of $275-300 \mathrm{~g}$ and were maintained at these weights throughout the experiment.

Apparatus. The apparatus consisted of four identical leverboxes that were housed in separate ventilated, sound-attenuating plywood chambers. The end walls and the rear walls of the boxes were made of sheet metal and the front wall and ceiling were made of transparent Perspex. The internal dimensions of the boxes were $22 \times$ $20 \times 19 \mathrm{~cm}$ high. Located in one end, $7.5 \mathrm{~cm}$ above the grid floor, was a Ralph Gerbrands lever and the access hole to the food magazine of a Ralph Gerbrands pellet dispenser, which delivered 45$\mathrm{mg}$ Noyes food pellets. The leverboxes were illuminated by a very dim houselight (GE No. 182028 -V bulb in series with a 470- $\Omega$ resistance). The signal (light) was produced by switching the resistance out of the circuit and illuminating the houselight at its full intensity.

Procedure. The animals were initially placed in the leverboxes with the lever removed for one 30-min session during which approximately 30 food pellets were delivered according to a randomtme 1-min schedule. On the next day, the levers were replaced and each leverpress produced a pellet. The rats were placed in the boxes until they had earned 50 pellets according to this continuous reinforcement schedule. All subsequent experimental sessions lasted for $50 \mathrm{~min}$.

For the next 12 days, the animals were reinforced for pressing the lever according to a random-interval (RI) 1-min schedule. This RI schedule was produced by sampling the computer's random- 
probability generator once per second and setting up the contingency that the next response would be reinforced with a probability of $1 / 60$ each second. There was no attempt to enforce a maximum interval length on this schedule. Following this procedure, the rats were divided into three groups of 8 (matched for final RI response rate). Each group subsequently received four sessions of one of the three response-reduction procedures. Group Extinction received the traditional procedure in which responses were no longer reinforced and no food was presented. For Group Free Food, responses also no longer produced food, but noncontingent food was delivered according to a variable time (VT) 1 -min schedule (minimum food-food interval $=4 \mathrm{sec}$, maximum $=120 \mathrm{sec}$ ). Food delivery was independent of leverpressing. All animals that received food in this phase received the same repeating pseudorandom sequence of the 24 intervals in the schedule. Group Signaled Food received the same treatment as Group Free Food except that each food delivery was preceded by a 3-sec illumination of the houselight (hence the minimum food-light interval $=1 \mathrm{sec}$ ). On the final day of the experiment, all animals were shifted to the free-food treatment for one session.

Response Measures and Statistical Analysis. It is well known that, although individual animals become quite consistent in their rates of responding on $\mathrm{RI}$ schedules, there is very high intersubject variability. For instance, in the present experiment, the animals' response rates varied from 9 to 50 responses per minute ( $\mathrm{rsp} / \mathrm{min}$ ). Thus, most between-subject comparisons have very little power. Because of this, a measure called the extinction ratio was used to assess the relative rates of extinction. This measure compares each animal's rate of responding during the last baseline day (B) with the rate on each extinction day (E) according to the formula $\mathrm{E} /(\mathrm{E}+\mathrm{B})$. Responses during the signaled periods were excluded from the calculations of the rates in this and in all subsequent signaled groups. A ratio of 0.5 means no decline in responding, whereas a ratio of 0 means complete cessation of responding. In addition, the raw response rates were analyzed. These analyses never contradicted the analyses of ratios, but because the raw scores were both redundant and less powerful, they will not be reported here.

The extinction ratios were analyzed using split-plot analyses (i.e., two factors: one between-subjects, one within-subjects). If the interaction term was significant, one-way analyses of variance (ANOVAs) were calculated between treatments on each day. These one-way analyses used a between-groups error term calculated using only that day's data. All post hoc analyses used Rodger's (1967) method. This method is similar to that proposed by Scheffe (1953). It involves choosing an orthogonal set of comparisons and contrasts and comparing them against Rodger's, rather than Fisher's, $F$ ratio. This method does not involve pairwise comparisons. As a general rule, I use the most efficient set of comparisons and contrasts that are interpretable. It is possible to know what percentage of the variance between the means the reliable set of contrasts account for by adding the reported $F \mathrm{~s}$ together and comparing them with the significant $F$ of the one-way ANOVA that was used to justify them. The ratio of the sum of the significant $F$ s to the overall $F$ represents the proportion of the variance (mean square) of the means that is accounted for by the set. This procedure prevents the redundant use of the variance in making decisions. An apparent exception to this rule is that sometimes when the means are evenly distributed, the orthogonal set of contrasts appears to misrepresent the data. In this case, I report pairwise comparisons. I often report several of these comparisons, but it must be remembered that the smallest significant range could be considered the base of an orthogonal set, and claiming it to be reliable implies that all larger ranges are also reliable. A 5\% Type 1 error rate was chosen for all comparisons.

\section{Results}

The animals all learned to leverpress for food in a typical manner, and on the last day of RI training before the response-elimination phase, the groups' mean response rates varied from 32 to $34 \mathrm{rsp} / \mathrm{min}[F(2,21)=0.05]$.

The mean extinction ratios for 10 -min blocks throughout the extinction and test days for the three groups are shown in Figure 1. Clearly, all three procedures caused responding to decline. The free-food treatment produced the slowest decline, and signaling the food produced an intermediate rate of decline. In absolute response rates, Group Extinction declined from 16.3 to $0.6 \mathrm{rsp} / \mathrm{min}$, Group Free Food declined from 24 to $12 \mathrm{rsp} / \mathrm{min}$ and the signaled group declined from 17 to $4 \mathrm{rsp} / \mathrm{min}$. The statistical analyses carried out on the extinction ratios confirm that these differences are reliable. Due to an error, 4 of the animals in Group Extinction received free food commencing on Day 4 rather than on the test day (Day 5) as planned, so these scores were used as their scores for the test day. In addition, 4 animals in Group Free Food received extinction on Day 4, so these animals were eliminated from the analyses on Days 4 and 5 . Because of these errors, data from Day 4 were not included in the statistical analyses reported here. In the split-plot analysis of the first 3 extinction days, both main effects and the interaction were reliable $[F(2,21)=27.8, F(2,42)=170$, and $F(4,42)=7.46]$. The groups differed reliably on each of the 3 days [minimum $F(2,21)=5.83$ ] and on each of these days, Groups Extinction and Signaled Food had lower ratios than Group Free Food [minimum $F(2,21)=$ 5.68]. On Days 2 and 3, Group Signaled Food had reliably higher ratios than Group Extinction [minimum $F(2,21)=2.94]$.

Although this conclusion must be taken with some caution because of the experimental errors described above, the final data points of Figure 1 suggest that instituting free food after extinction caused a substantial increase in responding in Group Extinction. A split-plot analysis comparing the ratios from Day 3 with those of Day 5 confirms this. Both main effects and the interaction were significant $[F(2,21)=6.50, F(1,21)=10.7$, and $F(4,21)=$

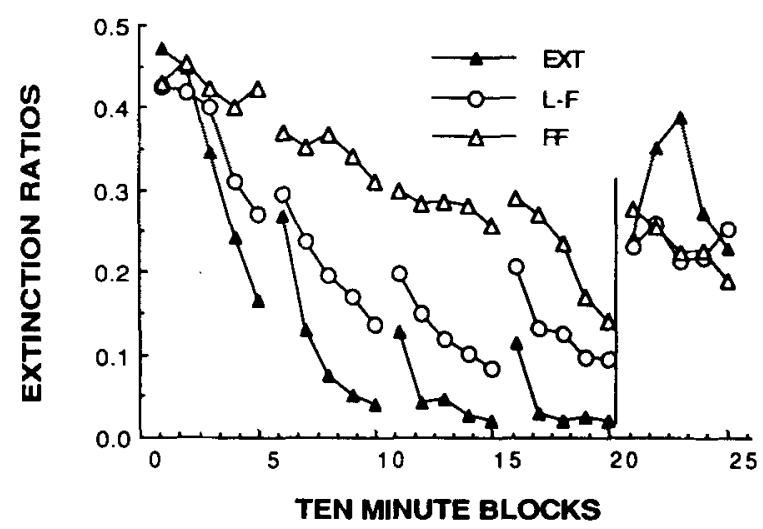

Figure 1. Extinction ratios in 10-min blocks [response rate/ (response rate + baseline response rate from the last random-interval day before extinction)] for the extinction days of Experiment 1 . There were five 10-min blocks per day. The gaps in the curves represent the boundaries between days. The vertical line divides the extinction days from the test day on which all groups received free food. 
$56.2]$. On Day 5, the groups differed $[F(2,21)=13.4]$, and on this day, Group Extinction was less suppressed than the other two groups $[F(2,21)=12.6]$. This contrasts with the earlier reported test for Day 3 , in which this group was the most suppressed group. There is also some evidence that introducing free food caused an increase in responding in Group Signaled Food: a relatedmeasures $t$ test comparing the last signaled-food day with the free-food day was reliable $[t(7)=3.10]$.

In addition to the daily analyses, for each of the first three sessions, the data were divided into 10-min blocks and analyzed (the subsequent days' analyses are not reported because of the error on Day 4). On each day, the groups started somewhat close together and then separated, with Group Free Food responding the most, Group Extinction responding the least, and Group Signaled Food responding at an intermediate rate. Split-plot analyses were carried out on these scores for the first 3 days of the extinction phase. Within the first 2 days, the scores essentially mirrored the daily analysis that was summarized earlier. Both main effects and the interactions of both analyses were reliable [minimum $F(2,21)=6.04, F(4.84)=$ 44.2 , and $F(8,84)=7.20$ ]. On Day 3 , the two main effects were reliable $[F(2,21)=26.5$, and $F(4,84)=13.3]$, but the interaction was not $[F(8,84)=1.75]$.

The main hypothesis that was being tested in this experiment is that free food produces contextual conditioning, and that this contextual conditioning facilitates responding. Signaling the free food reduces contextual conditioning because the signal becomes conditioned at the expense of the context. Until now, I have reported no independent evidence that the signal was conditioned. To do so, I collected the response rates during the signal in the signaled group, during the $3 \mathrm{sec}$ immediately preceding reinforcement in the free-food group, and during an equivalent period in the extinction group. These rates declined from $71.6 \mathrm{rsp} / \mathrm{min}$ on Day 1 to $40.4 \mathrm{rsp} / \mathrm{min}$ on Day 3 in Group Free Food, from 42.3 to $13.7 \mathrm{rsp} / \mathrm{min}$ in Group Signaled Food, and from 43.4 to $1.5 \mathrm{rsp} / \mathrm{min}$ in Group Extinction. Because the overall baseline response rates varied between groups during extinction, these scores (E) were converted to ratios using the withinsession response rates (B) for comparison, using the formula $E /(E+B)$. For Group Free Food, the scores varied from .50 to .53 , for Group Signaled Food, the rates varied from .37 to .34 , and, surprisingly, Group Extinction had scores of .49 and .47 , dropping to .22 on Day 3 . The scores differed significantly on Days 1 and $3\left[F_{\mathrm{s}}(2,21)=\right.$ $4.19,2.06$, and 3.63]. On Day 1, Group Signaled Food was more suppressed than the other groups $[F(2,21)=$ 3.88 , and on Day 3, Group Extinction was more suppressed than Group Free Food $[F(2,21)=3.55]$.

This relative suppression of responding during a short $\mathrm{CS}$ in the signaled group is not surprising. Short appetitive CSs often suppress appetitive instrumental behavior (e.g., see Karpicke, 1978), whereas longer ones sometimes enhance it (cf. Meltzer \& Hamm, 1974; Miczek \& Grossman, 1971). The low ratios in Group Extinction on Day 3 were simply a consequence of the overall low level of responding in that group. The low rate combined with the short amount of time sampled simply produced many ratios of zero because none of the relatively few responses occurred during this period.

\section{Discussion}

The results of Experiment 1 were straightforward. As in Rescorla and Skucy's (1969) experiment, free food significantly retarded the extinction of leverpressing. In addition, the present results replicated Rescorla and Skucy's finding that instituting noncontingent food after the extinction procedure produces an increase in responding. There was also somewhat weaker evidence that these unsignaled food presentations brought about an increase in responding in the signaled-food animals as well.

The novel result of this experiment is that signaling the food in a free-food procedure reduced the interference with extinction produced by noncontingent food. This result is similar to that of an experiment done by Pearce and Hall (1979), in which they found that off-baseline presentation of food later facilitated responding in extinction, and that signaling this food reduced the facilitation. Both results are consistent with the hypothesis that one of the causes of the interference with extinction is that the free food does not allow the context to extinguish and, to the extent that this contextual conditioning controls responding, the extinction of leverpressing is slowed. If the signal overshadowed contextual conditioning because it was a more contiguous predictor of the food than was the context, then the extinction process might have been facilitated.

The present results are also consistent with a general version of the generalization-decrement hypothesis, which would claim that, when a response-reinforcer contingency is removed, the rate of response reduction will be faster the more discriminable the change in the stimulus characteristics of the experiment. Traditional extinction generally produces fairly rapid response reduction because this procedure is quite discriminable from training-food is discontinued. On the other hand, the free-food procedure is much more similar to training than is the extinction procedure, thus responding is more persistent. The signaledfood procedure would appear to be more discriminable from training than the free-food procedure-the signal was added-but less so than extinction-the food was not discontinued. Several demonstrations that adding a responsecontingent stimulus facilitates extinction are consistent with this idea that a signal alone might facilitate extinction (e.g., Hurwitz \& Cutts, 1957; Melching, 1954).

\section{EXPERIMENT 2}

One way to determine whether signaling food reduces interference by increasing generalization decrement or by reducing contextual conditioning is to include a group that receives the signal in a manner that should not overshadow contextual conditioning. 
Experiment 2 was designed to equate the absolute stimulus differences between the signaled-food and the free-food procedures. The experiment included three groups. Groups Free Food and Forward received the same treatments as Groups Free Food and Signaled Food of Experiment 1. The third group (Group Backward) also received pairings of a 3-sec light with food except that for this group, the pairings were backward pairingsthat is, the light came on immediately after the food rather than before it.

The rationale was that the backward and forward treatments should be equally different from the training treatment, but only forward pairings should markedly reduce contextual conditioning. Thus, the generalizationdecrement hypothesis would predict that both procedures should reduce interference with response suppression. To the extent that the mechanism of the interference depends upon contextual conditioning, only the forward treatment should have a marked effect.

A second reason the backward-signaling procedure is of interest comes from some experiments reported by Fanselow (1980), who investigated the preference for signaled shock phenomenon. In those experiments, a conditioned inhibitor produced the opposite effect that forward signals did, suggesting that although conditioned excitors reduce contextual conditioning, conditioned inhibitors might enhance it. It is known that backward pairings of a signal and a reinforcer can sometimes produce conditioned inhibition (see Moscovitch \& LoLordo, 1968). If contextual conditioning controls at least some of the responding in the extinction of instrumental responding, and if the backward signals become inhibitory and thus enhance contextual conditioning, then the backward signals might enhance rather than retard the resistance to extinction produced by free food. Such a counterintuitive result would surely strengthen the argument that the context is involved in the control of instrumental responding and would weaken the simple generalization-decrement alternative.

\section{Method}

Subjects. In this experiment, 24 male Wistar rats obtained from the same supplier and maintained under the same conditions as those used in Experiment 1 were used. These rats, however, were not experimentally naive. They had served in a latent-inhibition experiment in which they had been exposed to varying numbers of 90 -sec clicker presentations and each rat had received eight pairings of the clicker with a $0.8-\mathrm{mA}$ electric shock. Before the treatment phase of the present experiment, the animals were reassigned to three groups, counterbalanced as much as possible for previous treatment, and were then given 7 days of RI training before the treatment phase. This gave them a total of 23 days of RI training, including the conditioning phase of the latent-inhibition experiment.

Apparatus. The apparatus consisted of four Campden Instruments leverboxes contained in sound- and light-attenuating enclosures. Although these boxes were two-lever chambers, only the left lever was present. The only other modification to the boxes was that the metal ceilings had been replaced by transparent Perspex ceilings, although the standard 28-V houselight still protruded through the ceilings. This houselight was normally illuminated very dimly (in series with a $470-\Omega$ resistance), but for the 3 -sec light stimulus, it was illuminated at its normal intensity. Food reinforcement again consisted of 45-mg Noyes food pellets.
Procedure. Following the RI training described above, the animals were exposed to one of three response-reduction procedures. For all three groups, the response-food contingency was removed (leverpresses ceased to result in food deliveries), but the animals continued to receive noncontingent food according to a VT1-min schedule. Group Free Food received unsignaled food. Group Forward's food deliveries were preceded by 3-sec illuminations of the light. Group Backward's food deliveries were immediately followed by 3 -sec light illuminations.

All groups received exactly the same schedule of food presentations. The minimum interfood interval was $4 \mathrm{sec}$ and the maximum was $120 \mathrm{sec}$. Thus, the minimum food-signal interval for Group Forward was $1 \mathrm{sec}$. All sessions were $50 \mathrm{~min}$ in duration. Following 6 days of the three treatments, all groups received 1 day of unsignaled noncontingent food (the free-food treatment).

\section{Results}

Because the groups had had extended RI training, they had quite high, but equivalent, rates of responding on the last baseline day before extinction [range of means = 59-64 rsp/min, range of individual response rates $=$ $20-111 \mathrm{rsp} / \mathrm{min} ; F(2,21)=0.092$ ].

The various groups' extinction ratios, divided into 10-min blocks, are shown in Figure 2. The impression given by this figure is that Group Forward suppressed responding faster and that Group Backward suppressed somewhat more slowly than Group Free Food. The absolute response rates of Group Forward declined from 39 to $6 \mathrm{rsp} / \mathrm{min}$ over the extinction days, whereas Group Free Food declined from 47 to $20 \mathrm{rsp} / \mathrm{min}$ and Group Backward declined from 53 to $22 \mathrm{rsp} / \mathrm{min}$. A split-plot anal$y$ sis on the daily mean extinction ratios of the 6 extinction days was carried out. Both of the main effects and the interaction were reliable $[F(2,21)=19.3, F(5,105)=$ 62.0 , and $F(10,105)=3.41]$. One-way ANOVAs were carried out on each day's data and all were reliable [minimum $F(2,21)=5.60]$, except for the final day $[F(2,21)$ $=2.91 \mathrm{~J}$. On each of the first 6 days, Group Forward was more suppressed than Group Backward [minimum $F(2,21)=5.37]$. Group Forward was more suppressed than Group Free Food on Days 2-5 [minimum $F(2,21)$ $=7.86]$. Finally, Group Backward was reliably less sup-

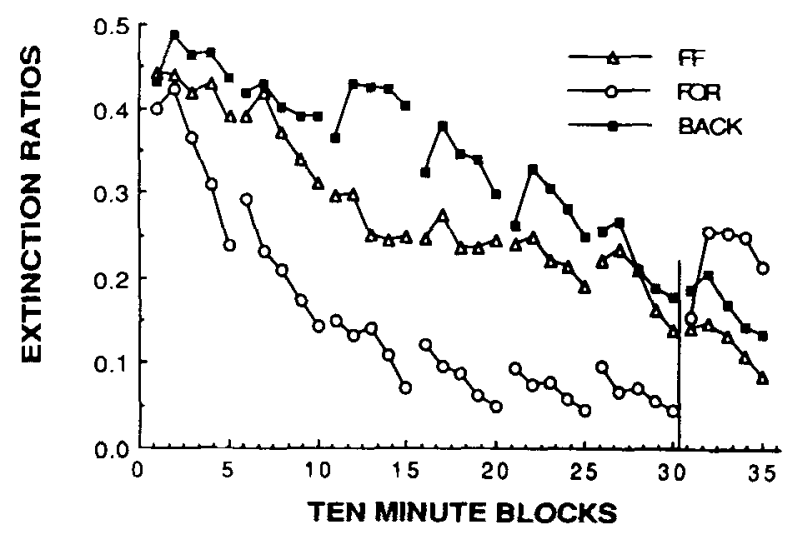

Figure 2. Extinction ratios for Experiment 2 reported over 10-min blocks. The gaps represent boundaries between days and the vertical line separates the 6 extinction days from the test day on which all animals received unsignaled free food. 
pressed than Group Free Food on the Extinction Day 3 $[F(2,21)=6.81]$. This difference represented nearly a twofold difference in absolute responding between the groups on this day ( $43 \mathrm{vs} .25 \mathrm{rsp} / \mathrm{min}$ ).

Figure 2 also shows that instituting unsignaled free food on the final day caused a fairly large increase in responding in Group Forward but no increase in the other two groups. A split-plot analysis comparing this day with the day preceding it confirms this. Only the interaction was reliable $[F \mathrm{~s}(2,21)=0.37, F(1,21)=1.22$, and $F(2,21)$ $=6.00]$. The between-groups analyses on both days were not significant $[F \mathrm{~s}(2,21)=2.91$ and 1.20]. Relatedmeasures analyses comparing each group's scores on the 2 days showed that Group Forward increased responding significantly, Group Backward did not change over the 2 days, and Group Free Food showed a reliable decline $[F \mathrm{~s}(1,7)=39.0,0.00$, and 9.52 , respectively $]$.

The analyses already described dealt with the daily means. Some analyses of the 10-min blocked data were also done. Split-plot analyses of the five daily $10-\mathrm{min}$ blocks were carried out for the first 3 days. The results paralleled the overall analysis described earlier. Both main effects and the interaction were reliable on each day [minimum $F(2,21)=6.12, F(4,84)=3.00$, and $F(8,84)=$ $2.61]$. A posteriori analyses essentially replicated the results of the daily analyses: Group Forward was more suppressed than Group Free Food on Days 2 and 3, whereas Group Backward was less suppressed than Group Free Food on only Day 3.

\section{Discussion}

The results of this experiment replicate the novel finding of Experiment 1. The animals exposed to signaled noncontingent food suppressed responding more rapidly than the animals exposed to unsignaled noncontingent food.

A more suprising finding concerns Group Backward. Not only did the food-light pairings fail to facilitate extinction relative to free food, but there was evidence that these pairings retarded response suppression. This result is inconsistent with the generalization-decrement hypothesis, which claims that the speed of extinction should depend on how discriminable the response-reduction procedure is from training. It seems reasonable to insist that the backward treatment should be more different from training than is the unsignaled free-food procedure, yet this treatment seemed to retard extinction even more than did unsignaled food. It would certainly be possible to claim that the backward treatment should produce less facilitation of extinction than the forward treatment because it may not be as discriminable from training. Such a claim could even be used to defend the position against a finding of no difference between Groups Free Food and Backward, but it would certainly be difficult to come up with an explanation of the present results in terms of generalization decrement in which the backward group responded more than Group Free Food.

The finding that the backward treatment seemed to retard response suppression is reminiscent of certain results reported by Wagner and Rescorla (1972). In those experiments, pairing an inhibitory stimulus with a neutral stimulus in a compound and then reinforcing the compound enhanced excitatory conditioning of the neutral stimulus. Furthermore, Fanselow (1980) has provided evidence that aninals find a context that has been paired with an inhibitor for shock more aversive than a control context in which the animals received unsignaled shocks. It has been established that backward pairings of a signal and a reinforcer often cause the signal to become a Pavlovian inhibitor (Moscovitch \& LoLordo, 1968). In the present experiment, it is possible that the backward stimulus became a Pavlovian inhibitor. If, as the associative argument claims, contextual conditioning plays a role in the control of responding, then it is possible that the presence of an inhibitor might increase contextual conditioning and hence further retard extinction.

As a second possible mechanism, backward conditioning might protect the context from the negative aftereffects of food delivery. Contemporary theorists have claimed (see Solomon \& Corbitt, 1974; Schull, 1979; Wagner \& Larew, 1985) that every affective event has a negative aftereffect, and this aftereffect can influence behavior in the opposite direction of the conventional affective direction of the event. Thus, it is possible that the presentation of food in a context causes that context to become excitatory, but in addition, the context, unlike a more traditional CS, continues on after the reinforcer has been terminated and therefore would be paired with the negative aftereffects as well. These pairings should weaken excitatory contextual conditioning. To the extent that a backward signal could overshadow the context and come to control these negative aftereffects, it should enhance excitatory contextual conditioning and thus prolong responding in extinction.

It should be remembered that in spite of the rather speculative nature of the alternative mechanisms that have been proposed for the enhancement of responding during extinction, the basic empirical result of the present experiment is inconsistent with the generalization-decrement hypothesis.

\section{EXPERIMENT 3}

Experiments 1 and 2 demonstrated that free food retarded the course of extinction, that signaling the food reduced the retardation, and that a backward signal enhanced the retardation. I have argued that a major determinant of performance during extinction may be contextual conditioning and that these treatments might have their differential effects on extinction because they affect contextual conditioning. Compelling though this argument might be, the initial experiments provided little independent evidence that the free-food procedure or the various signaling manipulations actually influenced contextual conditioning. Experiment 3 was designed to provide some such evidence.

I took advantage of an observation made by Rescorla and Skucy (1969) who, as a control procedure, gave non- 
contingent food to rats that had had no previous leverpressing training. They found a high and sustained "operant level" of leverpressing throughout the experiment. It is possible that this leverpressing might serve as a convenient measure of contextual conditioning, perhaps because it might indirectly monitor activity. Accordingly, in the present experiment, I included three groups of rats that never received a response-food contingency. They were initially magazine-trained and then received exposure to noncontingent food with the lever present. One of these groups received unsignaled free food (Group Free Food), a second group received the food signaled by the 3-seclight stimulus used earlier (Group Forward), and the third group received backward pairings of the light and food (Group Backward). A fourth group received RI training followed by standard extinction (no food) and then a test in which unsignaled food was presented (Group RI). This group was included to provide an estimate of the actual operant level of responding following extinction with which to compare the rates in the noncontingent groups and as a control to see whether the enhancement of responding following the reinstatement of food exceeds the operant, or baseline, level of responding elicited by free food in the various noncontingent groups.

\section{Method}

Subjects and Apparatus. Four groups of 8 naive male Long Evans hooded rats whose initial weights varied from $275-300 \mathrm{~g}$ were used in this experiment. Throughout the experiment, the rats were maintained at $80 \%$ of their initial weights. The apparatus and stimuli described in Experiment 1 were used.

Procedure. All animals received one initial 30-min session of magazine training during which the levers were removed and food pellets were delivered according to a random-time 1-min reinforcement schedule. On the next day, the animals in Group RI were shaped to press the levers for food reinforcement. On this day, the other three groups of animals remained in their home cages.

For the remaining 26 days of the experiment, all sessions lasted for 50 min. During each session, noncontingent food, programmed according to a VT 1-min schedule, was presented to three of the groups. For Group Free Food, these food presentations were not signaled, for Group Forward the food was signaled by a 3-sec increase in the intensity of the houselight, and for Group Backward the food presentations were immediately followed by the light.

For Group RI, the experiment was divided into three phases lasting 8,10 , and 8 days. In the initial baseline phase, these animals were reinforced for leverpressing according to an RI 1-min schedule. In the subsequent extinction phase, conditioning was extinguished; that is, the levers remained present but no food was delivered. In the final, test, phase of the experiment, the animals were presented with unsignaled noncontingent food on a VT 1-min schedule.

Response Measures and Analyses. Responding during each session and during the $3 \mathrm{sec}$ preceding the food presentations was recorded. Because there was no appropriate baseline for calculating extinction ratios and because of the widely differing rates of responding between the groups, the rates were all transformed into logarithms (i.e., $\log +1$ transform). These data are exclusively reported here. The statistical analyses and rejection criteria (alpha $=.05$ ) described earlier were again used here.

\section{Results}

The logarithms of mean daily response rates, blocked over 2 days, are shown in Figure 3. A split-plot analysis was carried out on each of these scores for each phase of the experiment. Figure 3 indicates that during the baseline phase, Group RI (mean $=22 \mathrm{rsp} / \mathrm{min}$ ) responded more than the other groups and that Group Backward (2.5 rsp/min) seemed to respond more than Groups Free Food (1.4 rsp/min) and Forward (0.79 rsp/min). The statistical analyses partially confirm this impression. They indicate that Group RI responded at a higher rate than the other three groups, and that Group Backward responded more rapidly than Group Forward. Group Free Food, however, did not differ reliably from Group Forward or Group Backward. In the analysis, the main effect for treatments was reliable $[F(3,28)=22.9]$, as was the main effect for trials $[F(3,17)=3.17]$; the interaction was not reliable $[F(9,84)=1.37]$. A posteriori tests indicated that Group RI responded more than the mean of the other three groups $[F(3,28)=19.89]$, and that Groups Forward and Backward differed $[F(3,28)=2.92]$. Group Free Food differed from neither Group Forward nor Group Backward.

During extinction, responding in Group RI declined (from 18 to $0.38 \mathrm{rsp} / \mathrm{min}$ ) and ultimately became less than that of Groups Free Food (2.7 rsp/min) and Backward (3.7 rsp/min). In this phase, Groups Backward and Free Food responded at a higher rate than Group Forward ( $0.56 \mathrm{rsp} / \mathrm{min}$ ). This impression is also supported by the analyses. Both main effects and the interaction were reliable $[F(3,28)=3.23, F(4,112)=9.16$, and $F(12,112)=$ 18.6]. The one-way analyses carried out between treatments on each extinction block were all significant [minimum $F(3,28)=2.47]$. On Day 1 of extinction, Group RI differed from the mean of the other groups $[F(3,28)=$ 8.84], and none of the other groups differed reliably. On Day 2, the combined mean of Groups Backward and RI differed from Group Forward $[F(3,28)=2.32]$. On Day 3, the mean of Group Forward was lower than the combined mean of the other groups $[F(3,28)=3.51]$. On the final 2 test days, the combined mean of Groups Forward and RI was lower than that of Groups Free Food and Backward [minimum $F(3,28)=3.46$ ]

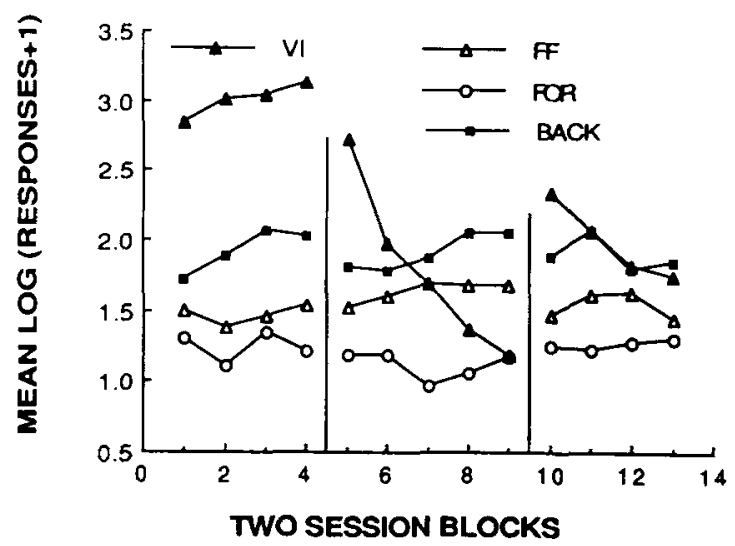

Figure 3. Mean log response rates for the four groups of Experiment 3. The vertical lines separate the training, extinction, and freefood phases for Group RI. The three noncontingent groups received the same treatments throughout. The data are reported in two-session blocks. 
It is fairly clear from Figure 3 that on the final test phase of the experiment, the three noncontingent food groups continued on much as they had throughout the experiment, but that Group RI, which had unsignaled food added, showed a marked increase in responding on Day 1 of the test. A split-plot analysis comparing the last day of the extinction phase with the first test day confirms this. The main effect for trials $[F(1,28)=9.04]$ and the interaction $[F(3,28)=19.6]$ were both reliable, but the main effect for treatment was not $[F(3,28)=2.20]$. Oneway ANOVAs within groups were carried out and only Group RI $[F(1,7)=21.5]$ showed a reliable change between the extinction and test days [from 0.38 to $14 \mathrm{rsp} / \mathrm{min}$; maximum $F(1,7)=4.94$ for the other groups]. In a final analysis carried out over the four test blocks, both the main effect for trials and the interaction were reliable $[F(3,84)=5.82$, and $F(9,84)=5.22$, respectively], but the treatment effect was not $[F(3,28)=$ 1.79]. During this phase, the one-way ANOVAs on Days 1 and 2 were reliable [minimum $F(3,28)=2.58$ ] . A posteriori tests indicated that on Day 1 , the mean of Group RI was higher than the combined mean of Groups Free Food and Forward $[F(3,28)=3.55]$, and on Day 2, the combined mean of Groups Backward and RI was higher than that of Group Forward $[F(3,28)=2.44]$.

In addition to the analysis of the overall response rates, responding during the $3 \mathrm{sec}$ before the food presentation was analyzed for the three noncontingent food groups. These data were blocked over the three phases of the experiment in order to obtain a large enough sample. Because the groups' baseline levels of responding differed, I did not analyze the raw log response rates but constructed ratios using the daily baseline scores (B) and the level of responding during the $3 \mathrm{sec}$ before food (CS) using the formula Ratio $=\mathrm{CS} /(\mathrm{CS}+\mathrm{B})$. These scores are shown for the three groups and the three phases in Figure 4. In an ANOVA carried out over the three phases of the experiment, only the main effect for groups was reliable $[F(2,21)=26.4, F(2,42)=.643$, and $F(4,42)=.917]$. A posteriori tests indicated that the mean ratio for Group Forward was much lower than those for the other two groups $[F(2,21)=25.1]$.

\section{Discussion}

The results of this experiment provide considerable support for the hypothesis that the signaling manipulation differentially affected contextual conditioning and that the signal itself was an effective stimulus. The three noncontingent groups showed differential levels of barpressing even though there was no response-food or temporal lever-food contingency except that the lever was, of course, one of the contextual cues. Thus, it would seem appropriate to consider leverpressing as a measure of contextual conditioning, perhaps because it was an indirect measure of activity. This is interesting because although it is fairly easy to demonstrate the effect of a signal on

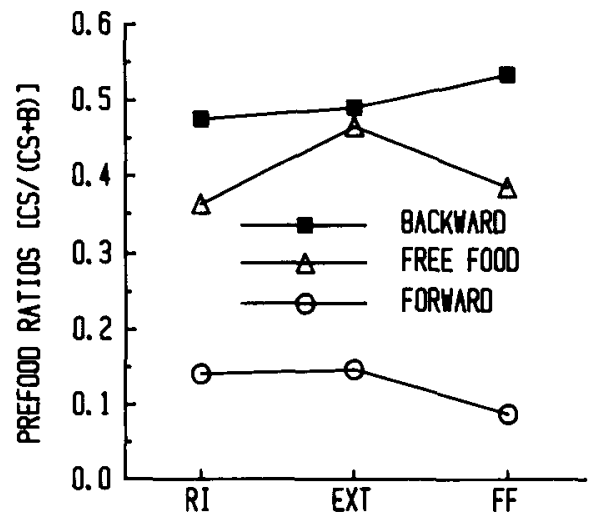

Figure 4. Prefood ratios for the prefood periods for the three noncontingent food groups of Experiment 3. The ratios were calculated using the formula [CS/(CS+B], in which $\mathrm{CS}$ is the number of responses during the $\mathrm{CS}$ periods for Group Forward and $\mathrm{B}$ is the baseline level of responding on each day. For Groups Backward and Free Food, CS is the number of responses from the $3 \mathrm{sec}$ immediately preceding food presentations. The three points in each function represent the random-interval (RI), extinction (EXT), and freefood (FF) stages for the extinction group.

contextual conditioning directly in aversive conditioning using a number of measures (e.g., see Baker et al., 1981; Bouton, 1984; Odling-Smee, 1978), it has not been so easy to do so in appetitive preparations using activity as a measure (e.g., see Balsam \& Gibbon, 1988; Rescorla et al., 1985; although see Grau \& Rescorla, 1984, for a successful demonstration). Hence, at the very least, the present experiment strengthens the case that signaling free food reduces contextual conditioning in an appetitive paradigm. In addition, these data are also consistent with the argument that the backward procedure actually enhanced contextual conditioning although the statistical support for this contention is not as strong.

The data from the period immediately preceding the food deliveries are relevant to the contention that the signal was an effective CS. Figure 4 shows that the animals in Group Forward rarely responded during this period, which coincided with the presentation of the CS. Although I made no behavioral observations of the animals, this pattern of responding is certainly consistent with the claim that the animals in this group approached the food magazine during the signal (see Karpicke, 1978; Karpicke, Christoph, Peterson, \& Hearst, 1977). The other two groups showed little evidence of any change in their leverpressing during this period.

The results of Group RI are of considerable interest. During the extinction period, this group provided evidence to support the claim that the differences found between the noncontingent groups are due to an enhancement of responding in Groups Free Food and Backward and not to a suppression of operant level of responding in Group Forward. Group RI's level of responding in this phaseduring which these animals received no food-was lower 
than those of Groups Free Food and Backward, even though Group RI had previously been exposed to a response contingency and had had a greatly enhanced level of leverpressing.

\section{GENERAL DISCUSSION}

The three experiments that I have reported here strongly support the role of the experimental context in extinction. Experiment 1 showed that free food retarded extinction and that signaling the food reduced that retardation. Experiment 2 replicated the critical result of Experiment 1 and produced the rather counterintuitive finding that a backward signal not only did not reduce the interference caused by free food, but appeared to enhance it.

I would argue that this constellation of findings suggests that conditioning of the context is deeply involved in the maintenance of responding in instrumental conditioning. During extinction, free food maintains contextual conditioning and thus in some way energizes responding, perhaps because it has become an "occasion-setter" during training (see Holland, 1983; Ross \& LoLordo, 1987) or, more conventionally, because its motivational properties are involved in evoking the response. The signal reduces this responding because it overshadows the context and thus reduces contextual conditioning. The increased interference found with the backward signal rules out any simple stimulus-generalization decrement explanation of the results and, furthermore, implies that a backward CS may actually enhance contextual conditioning.

The results of the first two experiments were certainly consistent with the role of contextual conditioning in extinction (see also Pearce \& Hall, 1979), but they provided no independent evidence that the treatment actually influenced contextual conditioning. Experiment 3 provides this evidence. Although food was not contingent upon leverpressing, the different food-signal relationships came to control differential leverpressing. The backwardsignaling treatment produced a fairly high level of responding, and forward pairings produced significantly less. Free food produced an intermediate level of responding. These results accord well with the hypothesis that the treatments had differential effects on contextual conditioning. Zamble (1969) had also reported data that indicates that contextual conditioning may facilitate the acquisition of an instrumental contingency.

Although noncontingent leverpressing might simply be an assay of general activity, it will only assay the behavior of animals in close proximity to the lever. It is thus possible that the different contingencies that have been used in the present experiments may simply have caused the animals to differentially allocate their time to different parts of the chamber. For instance, the forward-signaled groups might have used the signal to initiate an approach to the magazine, so between signals they might have spent more time in other parts of the chamber. On the other hand, the animals with no explicit food predictor might have come to spend more time close to the magazine and thus to the lever as well, so they would contact the lever more often simply because it was there. These possibilities merit future investigation.

Although the results of the three experiments suggest that contextual conditioning is important in the maintenance of responding produced by free food, they are somewhat ambiguous with respect to the specific mechanism involved. It is possible that contextual conditioning acts directly on the central motivational state that produces reinforced responses. This increased motivation either directly or indirectly (through its role as a stimulus) generates extra responses in extinction. Alternatively, contextual conditioning may generate extra baseline responding, and these responses might add to the normal number of responses during extinction. Although the absolute number of responses produced by free food in extinction was much greater than that produced in the noncontingent experiment, this may have simply reflected the differential sensitivity of the very different baseline levels of responding.

There is another class of possible explanations of some of the present results, which uses the concept of "superstitious reinforcement" (Neuringer, 1970; Skinner, 1948). One argument posits that the interference with extinction found in Group Free Food results from chance pairings of the food with the relatively high rates of responding found early in extinction. These chance pairings "superstitiously" maintain responding. Because the forward signal causes the animal to stop responding during its presence, for whatever reason, it imposes an arbitrary delay between the responses and reinforcement and thus reduces the effect of any superstitious contingency, so responding drops off rather rapidly. An anonymous reviewer suggested another plausible explanation of the present results, which also uses the concept of superstitious reinforcement. According to this account, the retardation of extinction using free food occurs because of the chance responsereinforcer pairings. This retardation is reduced by the perfectly correlated signal because it blocks or overshadows the much weaker response-reinforcer chance contiguity. In support of this account, there are several experiments that show that a signal interpolated between the response and the reinforcer in VI schedules reduces the response rate, presumably because it overshadows the responsereinforcer contingency (e.g., Pearce \& Hall, 1978; St. Claire-Smith, 1979; Williams, 1982). These explanations seem to be appealingly parsimonious. There are, however, at least three arguments against them.

First, Rescorla and Skucy (1969) included a control group that received free food during extinction but in which each response delayed the food presentations so that no chance food-lever pairings could occur. Although this procedure changed the temporal ordering of responding during extinction, it did not reduce the total number of responses. Second, Pearce and Hall (1979) reported an experiment in which they compared the effects of signaled and unsignaled food on responding in extinction. In their experiment, the food was given in the conditioning cham- 
bers with the levers removed prior to extinction training, yet it still had smaller effects-although similar to the present procedure-on the rate to extinction. The food was delivered in the absence of the lever, so this quite clearly eliminated the possibility of chance food-response pairings. Finally, although superstitious-responding explanations might account for the differences between forwardsignaled and unsignaled food, it is difficult to see how they would account for the enhanced responding found with backward signals.

Noncontingent reinforcers have been shown in several other procedures to take apparently paradoxical roles in both Pavlovian and instrumental conditioning, sometimes enhancing and sometimes reducing performance. Exposing the animal to the US prior to Pavlovian conditioning retards acquisition (see Randich \& LoLordo, 1979), but signaling the reinforcers reduces the retardation; that is, it enhances performance (e.g., see Baker et al., 1981). When the reinforcer is presented during training, it interferes with both classical and instrumental conditioning, and signaling these reinforcers often reduces the interference (e.g., see Baker, Singh, \& Bindra, 1985; Dickinson \& Charnock, 1985; Durlach, 1983). I have shown here that free reinforcers increase responding in extinction and that signaling reduces this effect. In certain classical conditioning paradigms, such as autoshaping, free reinforcers seem to enhance extinction, but in others, such as conditioned suppression, free reinforcers seem to retard extinction (e.g., see Ayres \& DeCosta, 1971). Finally, the introduction of free reinforcers following extinction in both paradigms causes increased responding and, in classical conditioning at least, manipulations of possible context-US associations often modulate this effect (see Bouton, 1984; Rescorla \& Skucy, 1969; the present experiments).

Associative models account for these paradoxical results by allowing contextual conditioning to interact with either CS-US or response-reinforcer associations in several ways. Under some circumstances, the context competes with the response or the CS for associations. Thus, preconditioning the context blocks future conditioning of a discrete CS in the US preexposure effect (e.g., see Baker et al., 1981; Randich \& LoLordo, 1979). Likewise, contextual conditioning competes with the CS or the response when free reinforcers are imposed during classical or instrumental conditioning (e.g., see Dickinson \& Charnock, 1985; Durlach, 1983). During extinction, however, context-reinforcer associations are seen to summate with or in some other way strengthen the response-evoking capabilities of both CS-reinforcer and response-reinforcer associations (e.g., see Bouton, 1984; the present experiments).

None of the assumptions that are necessary for these paradoxical effects of contextual conditioning are particularly unreasonable. Nonetheless, it must be remembered that a set of rules needs to be devised to account for when context associations will have one effect and when they will have the other. This is by no means an impossible task, but it does weaken the vaunted parsimony of the classical associative approach.

Up to this point, I have ignored an alternative theoretical interpretation of the role of contextual conditioning. This view, which is best typified by scalar expectancy theory (see Balsam, 1985; Gibbon, 1977; Gibbon \& Balsam, 1981), states that many of the effects of added reinforcers and contextual conditioning on learning about discrete CSs are actually performance effects. According to this view, animals learn about the context and the CS independently, but their ultimate performance is determined by comparing the levels of conditioning to the context and the CS. One of the implications of this view is that signaling reinforcers should not interfere with contextual conditioning. And, in fact, these workers have provided some evidence that contextual conditioning is by no means universally reduced by signaling the reinforcer (see also Rescorla et al., 1985). Assuming the results of the current experiment are mediated by contextual conditioning, the difference between the effects of forward pairings, backward pairings, and unsignaled food are inconsistent with this view.

Another explanation of the role of free food in extinction is that during training, the food becomes a discriminative stimulus for the response (see Uhl \& Garcia, 1969). This argument would then claim that the free-food procedure retards extinction because of its discriminative properties. This argument begs the question of just what properties of the reinforcer actually act as the discriminative stimulus for the response. One possible mechanism would be that it conditions the context, and the stimulus properties of the conditioned context actually act as the discriminative stimulus. If this argument were accepted, this notion could account easily for the fact that forward signaling reduces the interference of extinction.

Regardless of the fate of the above arguments, it would be naive to argue that resistance to extinction is solely a function of context-reinforcer associations. The relatively large and immediate increases in responding caused when free food was introduced during extinction in the present extinction and signaled-food groups and following similar manipulations in Rescorla and Skucy's (1969) experiments show that there is more to the increased resistance to extinction than responding produced by contextual excitation. In addition, Colwill and Rescorla (1985) and Adams and Dickinson (1981) have presented much evidence that rats can maintain some sort of representation of the response-reinforcer association during instrumental conditioning. Finally, it is virtually impossible to rule out the possibility that at least some of the resistance to extinction is caused by the chance responsefood pairings that must occur in these experiments. Although the present experiments show that resistance to extinction is remarkably sensitive to contextual manipulations, there is more to it than that. The context does seem to have some direct associative power to energize responding but, in addition, the animals also seem to maintain memories of past events and contingencies and these memories influence responding. 


\section{REFERENCES}

Adams, C. D., Dickinson, A. (1981). Actions and habits: Variations in associative representations during instrumental training. In N. E. Spear \& R. R. Miller (Eds.), Information processing in animals: Memory mechanisms (pp. 143-165). Hillsdale, NJ: Erlbaum.

A rres, J. J. B., DECosta, M. J. (1971). The truly random control as an extinction procedure. Psychonomic Science, 24, 31-33.

Baker, A. G., Mercier, P. A., Gabel, J., a Baker, P. A. (1981). Contextual conditioning and the US preexposure effect in conditioned fear. Joumal of Experimental Psychology: Animal Behavior Processes, 7, $109-128$

Baker, A. G., Singh, M. \& Bindra, D. B. (1985). Some effects of contextual conditioning and US predictability on Pavlovian conditioning. In P. D. Balsam \& A. Tomie (Eds.), Context and conditioning (pp. 73-103). New York: Academic Press.

BALSAM, P. D. (1985). The functions of the context in learning and performance. In P. D. Balsam \& A. Tomie (Eds.), Context and conditioning (pp. 1-21). New York: Academic Press.

Balsam, P. D., Gibbon, J. G. (1988). Formation of tone-US associations does not interfere with the formation of context-US associations in pigeons. Joumal of Experimental Psychology: Animal Behavior Processes, 14, 401-412.

Bonkes, R. A. (1973). Response decrements produced by extinction and response-independent reinforcement. Journal of the Experimental Analysis of Behavior, 19, 293-302.

Bouton, M. E. (1984). Differential control by context in the inflation and reinstatement paradigms. Joumal of Experimental Psychology: Animal Behavior Processes, 10, 56-74.

CAPALDI, E. J. (1967). Effect of $\mathrm{N}$-length and number of different $\mathrm{N}$ lengths and number of reinforcements on extinction. Journal of $E x$ perimental Psychology, 68, 230-239.

Colwill, R. M., Rescorla, R. A. (1986). Associative structures in instrumental learning. In G. H. Bower (Ed.), The psychology of learning and motivation (Vol. 20, pp. 55-104). New York: Academic Press.

Dickinson, A., \&harnock, D. J. (1985). Contingency effects with maintained reinforcement. Quarterly Journal of Experimental Psychology, 37B, 397-416.

DURLACH, P. J. (1983). Effect of signaling intertrial unconditioned stimuli in autoshaping. Journal of Experimental Psychology: Animal Behavior Processes, 9, 374-379.

FANSELow, M. S. (1980). Signaled shock-free periods and preference for signaled shock. Journal of Experimental Psychology: Animal Behavior Processes, 6, 65-80.

GibBoN, J. (1977). Scalar expectancy theory and Weber's law in animal learning. Psychological Review, 84, 279-352.

GibBon, J., \& BALSAM, P. D. (1981). The spread of association in time. In C. M. Locurto, H. S. Terrace, \& J. Gibbon (Eds.), Autoshaping and conditioning theory (pp. 219-253). New York: Academic Press.

GraU, J. W., \& Rescorla, R. A. (1984). Role of context in autoshaping. Joumal of Experimental Psychology: Animal Behavior Processes, 10, 324-332.

HeRRNStein, R. J. (1966). Superstition: A corollary of the principle of operant conditioning. In W. K. Honig (Ed.), Operant behavior: Areas of research and application (pp. 33-51). New York: AppletonCentury-Crofts.

HerRnstein, R. J., Morse, W. H. (1957). Some effects of responseindependent reinforcement on maintained operant behavior. Journal of Comparative \& Physiological Psychology, 50, 461-467.

Holland, P. C. (1983). Occasion setting in Pavlovian feature positive discriminations. In M. L. Commons, R. J. Hermstein, \& A. R. Wagner (Eds.), Quantitative analyses of behavior: Discrimination processes (Vol. 4, pp. 223-345). New York: Ballinger.

HuRwITZ, H. M. B., CurTs, J. (1957). Discrimination and operant extinction. British Journal of Psychology, 48, 90-92.

Kamin, L. J. (1969). Selective association and conditioning. In N. J. Mackintosh \& W. K. Honig (Eds.), Fundamental issues in associative learning (pp. 42-64). Halifax, Nova Scotia: Dalhousie University Press.
KARPICKE, J. (1978). Directed approach responses and positive suppression in the rat. Animal Learning \& Behavior, 6, 216-224.

Karpicke, J., Christoph, G., Peterson, G., Hearst, E. (1977). Signal location and positive vs. negative conditioned suppression in the rat. Joumal of Experimental Psychology: Animal Behavior Processes, 3, 105-118.

Mackintosh, N. J. (1974). The psychology of animal learning. London: Academic Press.

Melching, W. H. (1954). The acquired reward value of an intermittently presented stimulus. Journal of Comparative \& Physiological Psychology, 47, 370-374.

Meltzer, D., Hamm, R. J. (1974). Conditioned enhancement as a function of the percentage of CS-US pairings and CS duration. Bulletin of the Psychonomic Sociery, 4, 467-470.

Miczek, K. A., Grossman, S. P. (1971). Positive conditioned suppresion: Effects of CS duration. Joumal of the Experimental Analyses of Behavior, 15, 243-247.

Moscovirch, A., \& LoLoRdo, V. M. (1968). Role of safety in the Pavlovian backward fear conditioning procedure. Journal of Comparative \& Physiological Psychology, 66, 673-678.

Neuringer, A. J. (1970). Superstitious keypecking after three responseproduced reinforcements. Journal of the Experimental Analysis of Behavior, 13, 127-134.

ODLING-SMEE, F. J. (1978). The overshadowing of background stimuli by an informative $C S$ in aversive Pavlovian conditioning with rats. Animal Learning \& Behavior, 6, 43-51.

PAvLov, I. P. (1927). Conditioned reflexes. Oxford: Oxford University Press

Pearce, J. M., \& Hall, G. (1978). Overshadowing of instrumental conditioning of a lever press by a more valid predictor of reinforcement. Journal of Experimental Psychology: Animal Behavior Processes, 4, 356-367.

Pearce, J. M., \& Hall, G. (1979). The influence of context-reinforcer associations on instrumental performance. Animal Learning \& Behavior, 7, 504-508.

RANDICH, A., LoLoRDo, V. M. (1979). Associative and nonassociative theories of the UCS preexposure phenomenon. Psychological Bulletin, 86, 523-548.

Rescorla, R. A. (1966). Predictability and number of pairings in Pavlovian fear conditioning. Psychonomic Science, 4, 383-384.

Rescorla, R. A., Durlach, P., a Grau, J. W. (1985). Contextual learning in Pavlovian conditioning. In P. D. Balsam \& A. Tomie (Eds.), Context and learning (pp. 23-56). Hillsdale, NJ: Erlbaum.

Rescorla, R. A. SkUCY, J. C. (1969). Effect of response-independent reinforcers during extinction. Journal of Comparative \& Physiological Psychology, 67, 381-389.

RoDger, R. S. (1967). Type II errors and their decision basis. British Joumal of Mathematical \& Statistical Psychology, 20, 187-204.

Ross, R. T., LoLoRDo, V. M. (1987). Evaluation of the relationship between occasion-setting and instrumental discriminative stimuli: A blocking analysis. Journal of Experimental Psychology: Animal Behavior Processes, 13, 3-16.

ST. ClaIRE-SMITH, R. (1979). The overshadowing of instrumental conditioning by a stimulus that predicts reinforcement better than the response. Animal Learning \& Behavior, 7, 224-228.

SCHEFFÉ, H. (1953). A method of judging all contrasts in the analysis of variance. Biometrika, 40, 87-104.

SCHULL, J. (1979). A conditioned opponent theory of Pavlovian conditioning and habituation. In G. H. Bower (Ed.), The psychology of learning and motivation (Vol. 13, pp. 52-90). New York: Academic Press.

SKINNER, B. F. (1948). Superstition in the pigeon. Joumal of Experimental Psychology, 38, 168-172.

Solomon, R. L., \& CoRbitT, J. D. (1974). An opponent process theory of motivation: 1. Temporal dynamics of affect. Psychological Review, 81, 119-145.

SPENCE, K. W. (1966). Cognitive and drive factors in the extinction of the conditioned eyeblink in humans. Psychological Review, 73, $445-458$

Uhl, C. N., \& Garcia, E. E. (1969). Comparison of omission with 
extinction in response elimination in rats. Journal of Comparative \& Physiological Psychology, 69, 554-562.

WAGNER, A. R., \& LAREW, M. B. (1985). Opponent processes and conditioned inhibition. In R. R. Miller \& N. E. Spear (Eds.), Information processing in animals: Conditioned inhibition (pp. 233-265). Hillsdale, NJ: Eribaum.

Wagner, A. R., Rescorla, R. A. (1972). Inhibition in Pavlovian conditioning: An application of a theory. In R. A. Boakes \& M. S. Halliday (Eds.), Classical conditioning (Vol. 2, pp. 64-99). New York: Appleton-Century-Crofts.
Wiluams, B. A. (1982). Blocking the response-reinforcer association. In A. R. Wagner, R. Herrnstein, \& M. L. Commons (Eds.), Quantitative analyses of behavior: Acquisition processes (Vol. 3, pp. 427 447)

ZAMBLE, E. (1969). Conditioned motivational patterns in instrumental responding in the rat. Journal of Comparative \& Physiological Psychology, 69, 536-543.

(Manuscript received January 5, 1989; revision accepted for publication May 23, 1989.) 